\title{
Isolation of Milk Oligosaccharides using Solid-Phase Extraction
}

\author{
Robert E. Ward* \\ Department of Nutrition and Food Sciences, Utah State University, Logan, UT, 84322, USA
}

\begin{abstract}
A method was developed to isolate mono- and disaccharide-free oligosaccharides from human and bovine milk using a combination of enzymatic digestion of lactose and solid-phase extraction. In the initial trial, $2.5 \mathrm{~g}$ of oligosaccharides were isolated from one liter of human milk. In subsequent trials this was increased to over $5 \mathrm{~g}$ of oligosaccharides per liter. Compared to filtration-based extraction methods, this procedure allows for further isolation of oligosaccharide fractions via modulation of the column rinsing solvent. Neutral monosaccharide composition of the oligosaccharide polymers was investigated using gas chromatographic analysis of the monosaccharides as alditol acetate derivatives. Results indicate oligosaccharides are approximately made up of $24 \%$ fucose, $41 \%$ galactose, $22 \%$ glucose and $13 \%$ glucosamine. Isolated bovine and human milk oligosaccharides were compared to lactose as fermentation substrates for Bifidobacterium longum biovar infantis. Lactose fermentation yielded the greatest production of biomass followed by bovine and human milk oligosaccharides.
\end{abstract}

\section{INTRODUCTION}

Human milk is rich in complex soluble carbohydrate polymers, and little is understood of the function of these molecules. Some analytical studies have indicated that there may be as much as $20 \mathrm{~g} / \mathrm{L}$ of oligosaccharides in breast milk which would make them the third most concentrated component after lactose and fat $[1,2]$. However, unlike lactose and fat, these molecules do not appear to provide energy to infants as they resist the action of human digestive enzymes [3, 4]. Most human milk oligosaccharides (HMO) are elongation products of lactose, and are synthesized from glucose, galactose, glucosamine, fucose and sialic acid [2]. In addition to being concentrated, HMO are also diverse and at least 200 individual structures have been detected using mass spectrometry [5]. The compositions of HMO are also different among women, and any proposed function they may serve must address this heterogeneity [6].

As the composition of milk is the product of millennia of selective pressure, it stands to reason that individual constituents, especially those present in abundant quantities, would be expected to provide some sort of benefit to the infant or the mother or they would tend to be selected against over subsequent generations [7]. Several interesting bioactivities have been attributed to HMO. For example, they antagonize the binding of some strains of bacteria to epithelial cells [8], and a large epidemiologic study indicated that sugar epitopes resulting from an individual genotype appear to protect against diarrhea in breast fed infants [9]. Furthermore, those containing sialic acid may serve as immune modulators $[10,11]$. It has also been suggested that HMO may serve as growth factors for colonic microbiota [2], as it has long been known that breast feeding results in increased levels of fecal bifidobacteria with respect to infant formula. Based on their structure and composition, HMO may affect

*Address correspondence to this author at the Department of Nutrition and Food Sciences, Utah State University, Logan, UT, 84322, USA;

Tel: +1 435797 2153; Fax: +1 435797 4758; E-mail: robert.ward@ usu.edu the gut microbiota consortium either by selectively providing growth factors and energy substrates to some members, or alternatively via binding and eliminating others, or both. However, to begin to address whether HMO might be modulating this activity, it is first necessary to isolate them in large enough quantities to serve as fermentation and or growth factor substrates in microbiological media, and to insure that they are entirely free of milk mono and disaccharides.

Prior to the initiation of this work, it was noted in the literature that several groups have undertaken the task of isolating HMO from other constituents in milk, although mostly for quantification as opposed to the production of substrates for biochemical assays. The general scheme has been to first remove the fat from milk by centrifugation, and then the protein by precipitation with organic solvents [12]. To separate the HMO from lactose, gel filtration has often been used. This is a slow method that requires long separation times and has a low capacity. In contrast, two methods have been published for obtaining HMO in large quantities, one using charcoal column chromatography [13], and the other nanofiltration [14]. In each case the milk fat was first removed using centrifugation, and protein precipitated with organic solvents. Both groups then enzymatically converted lactose to glucose and galactose to facilitate separation. Brand-Miller et al. [13] used charcoal column adsorption to retain oligosaccharides on the column, while monosaccharides were eluted. However, little methodological information was provided, and the resulting product was not well characterized. Using nanofiltration, Sarney et al. [14] isolated HMO from other milk constituents, and compared the resulting HMO produced with gel filtration. Their yield with nanofiltration was 6.7 grams of HMO from 1L of milk, yet there did appear to be residual lactose in the oligosaccharide fraction produced with nanofiltration, but not in that prepared using gel filtration. Nanofiltration is an attractive method for HMO isolation due to the speed with which separations can be performed and it does not require the use of organic solvents. Nonetheless, nanofiltration does not allow 
for further separation of oligosaccharide fractions and it is unclear if it can be effective in removing all mono and disaccharides.

This report details the development and optimization of an isolation scheme for HMO using solid-phase extraction with graphitized carbon. It is low cost and, allows for complete removal of lactose and monosaccharides, and can be further optimized for isolation of selected fractions of HMO.

\section{MATERIALS AND METHODOLOGY}

Unless otherwise noted, all chemicals were obtained from Thermo Fisher Scientific (Waltham, MA). In the development and optimization of this method, frozen, pooled human milk was provided by the Mother's Milk Bank of San Jose, CA, by Dr. Jimi Francis, University of Nevada, Reno and by Prolacta Bioscience. Milk samples were kept at $-80^{\circ} \mathrm{C}$ until use. A sialic-acid rich Bovine milk fraction was provided by Fonterra (Auckland, NZ).

The process for isolation of HMO from milk involves six steps which are outlined in Fig. (1). Milk is thawed at room temperature, well mixed, and centrifuged at $5000 \times \mathrm{g}$ for 30 min at $4^{\circ} \mathrm{C}$ (Step 1). Solid fat is removed from the top, and subsequently mixed with one volume of distilled water at $37^{\circ}$. The lipid/water suspension is gently mixed by swirling for several minutes, and centrifuged at $4{ }^{\circ} \mathrm{C}$. This is repeated a second time, and all 3 aqueous phases are pooled prior to the next step.

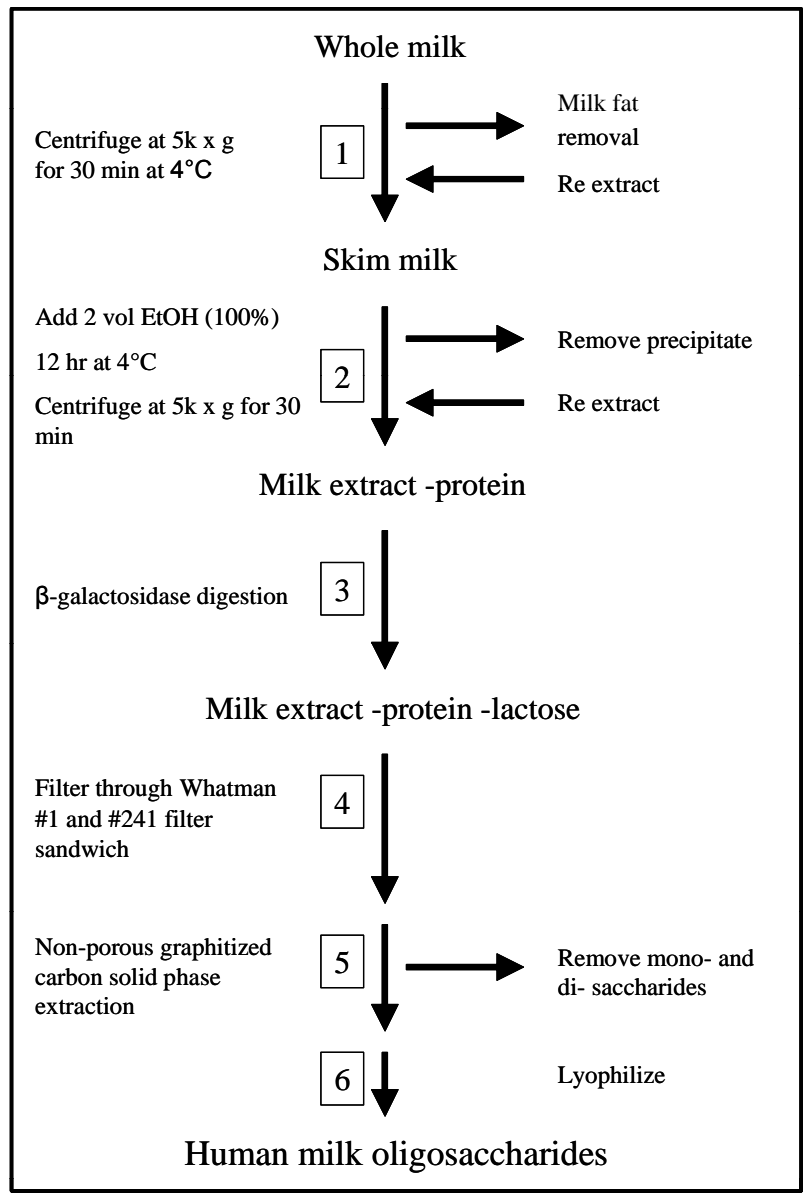

Fig. (1). Isolation scheme for obtaining mono and disaccharide free oligosaccharides from milk.
In Step 2, two volumes of ethanol are mixed with defatted milk, and kept overnight at $4^{\circ} \mathrm{C}$. This process precipitates protein, which is removed by centrifugation at $5000 \times \mathrm{g}$ for $30 \mathrm{~min}$ at $4^{\circ} \mathrm{C}$. The precipitate removed from the centrifuge tubes is resolubilized in two volumes of 2:1 ethanol:water, swirled to facilitate mixing, and then subjected to centrifugation at $5000 \mathrm{x} \mathrm{g}$ for 30 minutes at $4^{\circ} \mathrm{C}$. This is repeated a third time, the supernatants of the three precipitation/centrifugations are pooled, and ethanol removed by rotary evaporation.

To digest lactose (Step 3), milk extract is buffered by the addition of $40 \% \mathrm{v} / \mathrm{v} 0.123 \mathrm{M}$ phosphate buffer, $\mathrm{pH} 6.5$, an appropriate amount of $\beta$-galactosidase of Kluyveromyces fragilis (Sigma Aldrich, St. Louis, MO) is added based on the estimated lactose concentration and quoted enzyme activity, and the sample incubated for $3 \mathrm{~h}$ at $37^{\circ} \mathrm{C}$. After lactose digestion, particulates are removed via filtration with vacuum through a Whatman \#1 and \#541 filters in a series.

Monosaccharides and residual disaccharides are removed by solid-phase extraction using graphitized carbon columns (Supelco Inc., Bellefonte, PA) (Step 5). Prior to separation, cartridges are activated according to manufacture's protocol. A manifold holding 12 SPE columns (Fig. 2), each with a bed weight of 10 grams and $60 \mathrm{ml}$ volume, was adapted for large volumes by placing the manifold lid on a thin layer chromatography tank with the vacuum port located near the top. This allows for several column volumes to be pulled through the column without having to break the vacuum, and since the air intake valve is near the top of the tank there is room for a large volume of eluate to collect. Columns are loaded with 2 volumes of sample $(120 \mathrm{ml})$, rinsed with 2 volumes of deionized water, eluted with 2 volumes 60:40 acetonitrile/water containing $0.01 \%$ trifluouroacetic acid, and then rinsed with 2 volumes $\mathrm{ml}$ of deionized water. Using this protocol, it is possible to process one liter of milk in a few hours. Acetonitrile is removed with rotary evaporation, and the extract freeze dried.

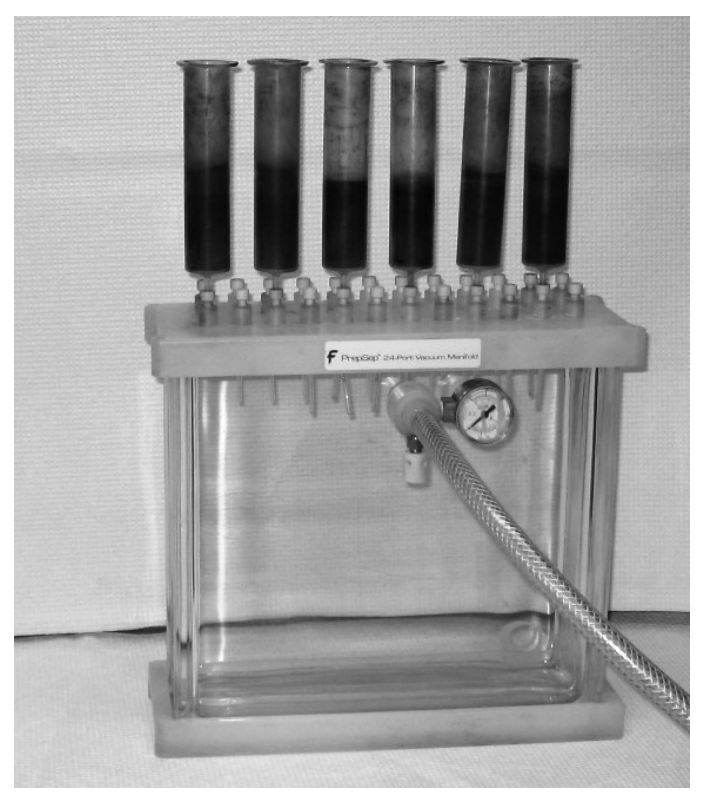

Fig. (2). Modified solid-phase extraction manifold for processing large volumes of milk. 
A similar strategy was utilized for isolation of oligosaccharides from a bovine colostrum powder. One hundred grams was diluted in $1 \mathrm{~L}$ deionized water, and otherwise the method was the same.

\section{Carbohydrate Measurements}

Where indicated, total carbohydrate was measured colorimetrically by the method of Dubois [15], and lactose and free glucose were measured in the HMO using an enzymatic kit (R-biopharm, So. Marshall, MI).

\section{High Performance Thin Layer Chromatography}

High performance Thin Layer Chromatography (HPTLC) was performed by the method described by Kunz et al. (1999) using $10 \times 20 \mathrm{~cm}$ plates from Whatman Ltd. ( Maidstone, UK). In short, samples containing oligosaccharides $(2-\mu 1)$ were spotted on plates and developed with a butanol-ethanol-water-acetic acid-pyridine (5:50:15:1.5:5, $\mathrm{v} / \mathrm{v} / \mathrm{v} / \mathrm{v} / \mathrm{v})$ solvent system. To detect carbohydrates, plates were sprayed with orcinol solution $\left(2 \mathrm{mg} / \mathrm{ml} 70 \% \mathrm{H}_{2} \mathrm{SO}_{4}\right)$ and placed in a $100^{\circ} \mathrm{C}$ oven for $15 \mathrm{~min}$.

\section{D SPE and HPTLC Separation}

The interaction of HMO with the graphitized carbon matrix of the SPE columns was investigated by loading a column (500 mg adsorbant, $6 \mathrm{ml}$ volume) with milk extract (post lactose digestion), and then eluting the adsorbed carbohydrates with an aqueous solution containing an increasing concentration of butanol. Oligosaccharides were eluted by sequentially rinsing the column with 3 volumes $(15 \mathrm{ml})$ of deionized water containing an increasing concentration of butanol. The percentage of butanol in the column rinse was $0.25 \%, 0.33 \%, 0.5 \%, 0.67 \%, 1 \%, 1.33 \%, 2 \%, 4 \%$. The last rinse contained $0.1 \%$ TFA in addition to the $4 \%$ butanol. The carbohydrate concentration of elution fractions was measured using the phenol-sulfuric acid method, and $5 \mathrm{ml}$ aliquots were lyophilized. Each fraction was brought up to a concentration of roughly $1.75 \%$ carbohydrate, $2 \mu \mathrm{l}$ aliquots were spotted on TLC plates, and were run according to standard procedure.

\section{Gas Chromatography of Alditol Acetate Derivatives}

The monosaccharide composition of HMO was determined by gas chromatography of alditol acetate derivatives. The general method for GC analysis was taken from York et al. [16]. In short, approximately $250 \mathrm{ng}$ of HMO was added to a $4 \mathrm{ml}$ vial and hydrolyzed by the addition of $250 \mu \mathrm{l}$ $2 \mathrm{M}$ triflouoroacetic acid (TFA) for $1 \mathrm{~h}$ at $121{ }^{\circ} \mathrm{C}$. Vials were cooled to room temperature, and TFA evaporated at $40{ }^{\circ} \mathrm{C}$ under a stream of nitrogen gas. To facilitate complete removal of TFA, $0.3 \mathrm{ml}$ of isopropyl alcohol was added and sample reevaporated. Monosaccharides were reduced by 250 $\mu 1 \mathrm{M}$ ammonia containing $10 \mathrm{mg} / \mathrm{ml}$ sodium borohydride, at room temperature for $1 \mathrm{~h}$. Excess borohydride was converted to borate by the addition of 2-3 drops of glacial acetic acid, and then $250 \mu \mathrm{l}$ acetic acid-methanol 1:9 (v/v) added to each vial and the resulting solution evaporated at $40{ }^{\circ} \mathrm{C}$ under nitrogen. Three additional evaporations were conducted in the same manner, and then three further evaporation were conducted with $250 \mu \mathrm{l}$ methanol. The alditols were then $\mathrm{O}$ acetylated by the addition of $50 \mu \mathrm{l}$ of acetic anhydride and $50 \mu \mathrm{l}$ pyridine, and incubated at $121{ }^{\circ} \mathrm{C}$ for 20 minutes. Vials were cooled, and $200 \mu 1$ of toluene added, and evaporated at room temperature. This was repeated a second time, and then the alditol acetates partitioned between $0.5 \mathrm{ml}$ of dicholormethane and $0.5 \mathrm{ml}$ of water. The water was then extracted two more times with $0.5 \mathrm{ml}$ dicholormethane, and the organic phases combined and dried. The samples were resolubilized in $100 \mu \mathrm{l}$ acetone, and transferred to a GC vial insert. Free monosaccharides were prepared in the same way, except the hydrolysis step with TFA was not performed.

Per-O-acetylated monosaccharides were analyzed on a Hewlett-Packard 6890 Series gas chromatograph equipped with a flame ionization detector (FID) and a $30 \mathrm{~m} \mathrm{DB}$ $225 \mathrm{MS}$ capillary column $(0.25 \mathrm{~mm}$ i.d.; $0.25 \mu \mathrm{m}$ film thickess). Hydrogen was used as the carrier gas at $36.5 \mathrm{ml}$ $\min ^{-1}$. Injector temperature was maintained at $225{ }^{\circ} \mathrm{C}$, and the split ratio was $1: 25$. Injection volume was $1 \mu 1$, oven temp was kept at $220{ }^{\circ} \mathrm{C}$ for entire run. Nitrogen was used as the make-up gas at $30 \mathrm{ml} / \mathrm{min}$, and detector was operated at $250{ }^{\circ} \mathrm{C}$. Peak identification was made by comparing reten-

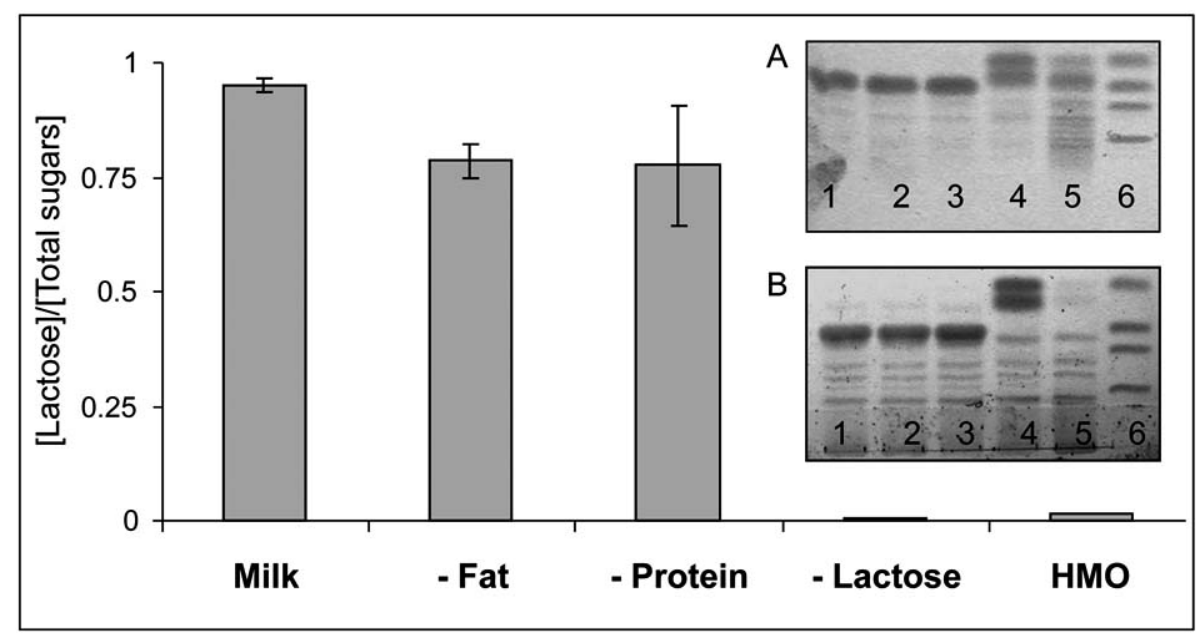

Fig. (3). Ratio of total carbohydrates to lactose in human milk fractions during oligosaccharide purification. Total sugars were measured colorimetrically and lactose was measured enzymatically. Insets A and B: HPTLC profiles of fractions from the initial and second purification 1) whole milk 2) skim milk 3) after protein precipitation 4) after lactose digestion 5) after solid phase extraction 6) standards (from top) glucose, lactose, raffinose, stachyose. 
tion time to individual monosaccharide standards deritivatized using the same process.

\section{Oligosaccharide Fermentation Assay}

The fermentation of human and bovine milk oligosaccharides was measured using Bifidobacterium longum b.v. infantis according to the method of Ward et al. [17]. In short, MRS media was made up with isolated oligosaccharides instead of glucose at $1 \%(\mathrm{~m} / \mathrm{v})$. Growth was measure with a Klett-Summerson colorimeter (Klett Manufacturing Co., Inc., New York, NY) at specific time points using the no. 45 (green filter).

\section{RESULTS}

In three subsequent extractions the yield of oligosaccharides per liter of breast milk was approximately $2.5 \mathrm{~g}, 5.5 \mathrm{~g}$, and 5.2 grams. The ratio of lactose (measured enzymatically) to total sugars (measured colometrically) at various stages in the procedure is shown in Fig. (3), and inset photos show HPTLC carbohydrate profiles resulting from these steps. It is worth noting when interpreting this figure, and the other data presented in this study, that of the five monosaccharide constituents of HMO, only glucose and galactose are detected by the colorimetric Dubois method, and only glucose, galactose and fucose stain with the orcinol reagent on TLC plates. From the figure it is clear that the majority of carbohydrate in breast milk is lactose, and approximately $5 \%$ of glucose and galactose is bound up in oligosaccharides which is consistent with previous estimates [10].

After the initial extraction with the $2.5 \mathrm{~g} / \mathrm{L}$ HMO yield, it was reasoned that the process might be improved, and changes to the process outlined in Fig. (1) included rextraction of the removed fat (Step 1) and protein (Step 2) to prevent any loss of HMO. Inset A of Fig. (3) shows the carbohydrate profiles after various stages of the first extraction, and from the image it appears there is some undigested lactose and some residual monosaccharides in the final HMO (Lane 5). For the second trial this was addressed by optimizing the enzymatic digestion of lactose, and modifying the SPE column loading protocol. According the manufacturer, $\beta$-galactosidase from Kluyveromyces fragilis has relatively sharp $\mathrm{pH}$ and temperature optima ( $\mathrm{pH} 6.5$ and $\left.37^{\circ} \mathrm{C}\right)$. Care was taken to insure that the reaction was conducted under these conditions, and the amount of enzyme added to the milk extract was doubled. In the second round of purification the progress of hydrolysis was monitored and determined to be complete after 3 hours (data not shown).

According to Redmond and Packer [18], the capacity of graphitized carbon SPE columns to adsorb the trisaccharide raffinose is approximately $27 \%$ of its mass. In the first trial, six columns, each with $10 \mathrm{~g}$ adsorbent beds and $60 \mathrm{ml}$ volumes, were used to extract $1 \mathrm{~L}$ of milk. The theoretical capacity of this system for oligosaccharides is over $16 \mathrm{~g}$, which is in excess of the level of HMO in milk, and yet only $2.5 \mathrm{~g}$ were isolated. To better understand the interaction of HMO with the adsorbent, a binding study was conducted with a small SPE column (500 mg bed, $6 \mathrm{ml}$ volume) and 5, 10, 20, 25 and $30 \mathrm{ml}$ of milk. The findings (data not shown) indicated that graphitized carbon SPE columns could bind up to approximately $20 \%$ of their weight in HMO, and all the $\mathrm{HMO}$ in the 5 and $10 \mathrm{ml}$ of milk. As the loading of the col- umn was further increased up $25 \mathrm{ml}$ of milk, no more than $20 \%$ of the weight of the column was bound, and the percentage bound per $\mathrm{ml}$ of milk went down. Thus, from $10 \mathrm{ml}$ of milk approximately $97 \mathrm{mg}$ of HMO were bound, and 101 mg were extracted from $25 \mathrm{ml}$ of milk. However, when 30 $\mathrm{ml}$ was applied to the column, only $39 \mathrm{mg}$ of HMO were adsorbed to the column, which indicates that overloading the column washes off HMO that had been previously bound. In the second and third trial this was addressed by increasing the number of columns on the manifold to 12 , by diluting the HMO extract prior to loading columns by doubling the volume, and by alternating rinse steps with deionized water in between column loadings. These changes led to a $50 \%$ increase in yield, however, it would appear that the overall yield could still be improved via a better understanding of the factors that limit HMO binding as column loading is increased.

To investigate the potential of this method to isolate specific fractions of HMO a second binding study was conducted with a small column (500 mg bed, $6 \mathrm{ml}$ volume). After loading the column, it was rinsed with solutions containing an increasing percentage of butanol to probe the interaction of the carbohydrates with the graphitized carbon adsorbent, and to get a better understand the size distribution of the HMO. This experiment was conducted with butanol, rather than acetonitrile, which was used in the bulk purification, as prior work had shown this solvent system to be effective in isolating specific oligosaccharides [18]. In Fig. (4), the carbohydrates in Lane 1 consist of the material that eluted from the column during the application of the sample. Comparing this lane to the standards at the other end of the plate indicates that the eluate consists primarily of glucose and galactose. Lane 2 contains material that was rinsed off the loaded column with water, and consisted primarily of monosaccharides, as well. Lanes 3-9 show the effects of increasing the concentration of butanol in the column rinse, and it is clear that as the concentration increases, different fractions of HMO are released. From this data it appears HMO elute in three broad groups, one near the top (Lanes 3-6) with a similar migration distance to the disaccharides lactose (Lane 11) and sucrose (Lane 12) and the trisaccharide raffinose, a second group (Lanes 3-8) with a similar mobility to the tetrasaccharide stachyose (Lane 12), and a group that remains at the origin (Lanes 6-10). On the bottom of the figure a bar graph of the relative concentration of sugars in each fraction is superimposed, and summing the contribution of Lanes 3-6 indicates that greater than $2 / 3$ of the HMO appear to be triand tetrasaccharides. This appears to be in agreement with the observation by LoCascio et al. [19] that B. longum bv. infantis preferentiall uses small HMO for fermentation substrates, which they find to represent $63.9 \%$ of the total. Starting in Lane 6, a band at the origin appears and becomes darker in the subsequent four fractions (Lanes 6-10). , and Lanes 9 and 10 consisted solely of this band. In Lane 10, $0.1 \%$ Trifluoroacetic acid (TFA) was added to the $4 \%$ butanol in the rinse. According to Redmond et al. [18], acidic sugars (including phosphorylated and sulfated monosaccharides) are strongly adsorbed to graphitized carbon, and will not be eluted by the addition of an organic modifier unless a volatile acid, such as TFA, is added. Thus, it would appear that the band at the bottom of Lane 10 represents acidic 
HMO, although this was not confirmed, and sialic acid is not visualized by the orcinol spray applied to the HPTLC plate.

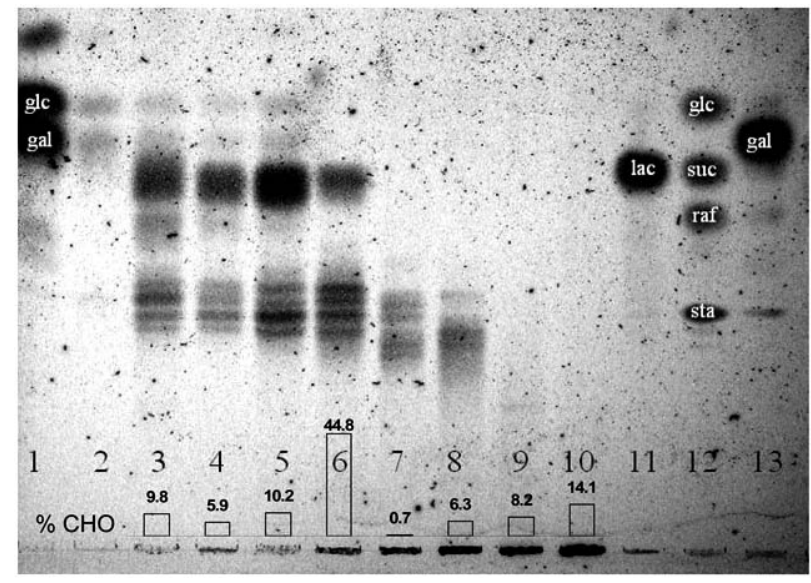

Fig. (4). HPTLC plate showing interaction of HMO with graphitized carbon as a function of elution solvent percentage. Lanes: 1) Eluate from column loading 2) column rinse with DI water 3) $0.25 \%$ butanol 4) $0.33 \%$ butanol 5) $0.5 \%$ butanol 6) $0.67 \%$ butanol 7) $1 \%$ butanol 8) $1.33 \%$ butanol 9) $2 \%$ butanol 10) $4 \%$ butanol $+0.1 \%$ TFA 11) lactose 12 ) from top: glucose, sucrose, raffinose, stachyose 13) galactose.

HMO isolated in Trial 1 and Trial 2 were characterized by gas chromatographic analysis of their constituent monosaccharides as alditol acetate derivatives, and the results are shown in Fig. (5). Data for sialic acid is not shown, as the method does not detect this monosaccharide. The first step in this procedure is to hydrolyze polymers to monomers using TFA, and by omitting this treatment free sugars were detected. Comparison of free and bound sugars confirms that HMO isolated in the first trial contained a significant proportion of free monosaccharides, and these were removed by modifications to the method in subsequent trials. In the inset table the percentage of bound monosaccharides in HMO from each trial is compared and the results show the composition to be very similar. To the best of the author's knowledge, this is the first such report on the monosaccharide composition of a bulk HMO isolate.

While it has long been speculated that HMO are used as energy sources for colonic microbiota, definitive evidence for this activity was only provided recently $[17,19,20]$. Bovine milk also contains oligosaccharides (BMO), yet they are present in much lower levels than in human milk. Nonetheless, due to the large volume of bovine milk produced each year, it was of interest to determine if these oligosaccharides are fermentable, and if so, how they compared to HMO as a substrate. To investigate this activity, they were isolated from a powdered milk fraction and used as the sole carbohydrate in an adapted MRS media. Due to the low yield, there was not sufficient BMO to conduct trials with multiple strains, so the bacterium with the highest activity on HMO, Bifidobacterium longum biovar infantis, was chosen to test this assay. The results of the growth assay is shown in Fig. (6), and it is clear that not only can B. longum bv. infantis use the BMO as an energy source, they appear to be more effective than HMO. There are several differences between $\mathrm{HMO}$ and BMO which might explain this finding. For example, fewer individual oligosaccharide species have been detected (40 for BMO vs. >200 for HMO), and over $70 \%$ contain at least one sialic acid [21] . Furthermore, in general they are dominated by tri- and tetrasaccharides, do not contain fucose, and the largest one detected to date is a heptamer [22]. Previous studies investigating prebiotic effects of HMO have indicated bifidobacteria preferentially consume small HMO as well as small oligofructose polymers $[19,20]$.

\section{DISCUSSION}

Although oliogsaccharides are a prominent component of human milk, this compositional feature is not shared among all mammals. Levels in ruminant milks are low [23], whereas primate milks typically contain more and more diverse oligosaccharides [24], as do elephants [25]. Nonetheless, there does not seem to be any clear indication of their functional importance that can be deduced from phylogenetic

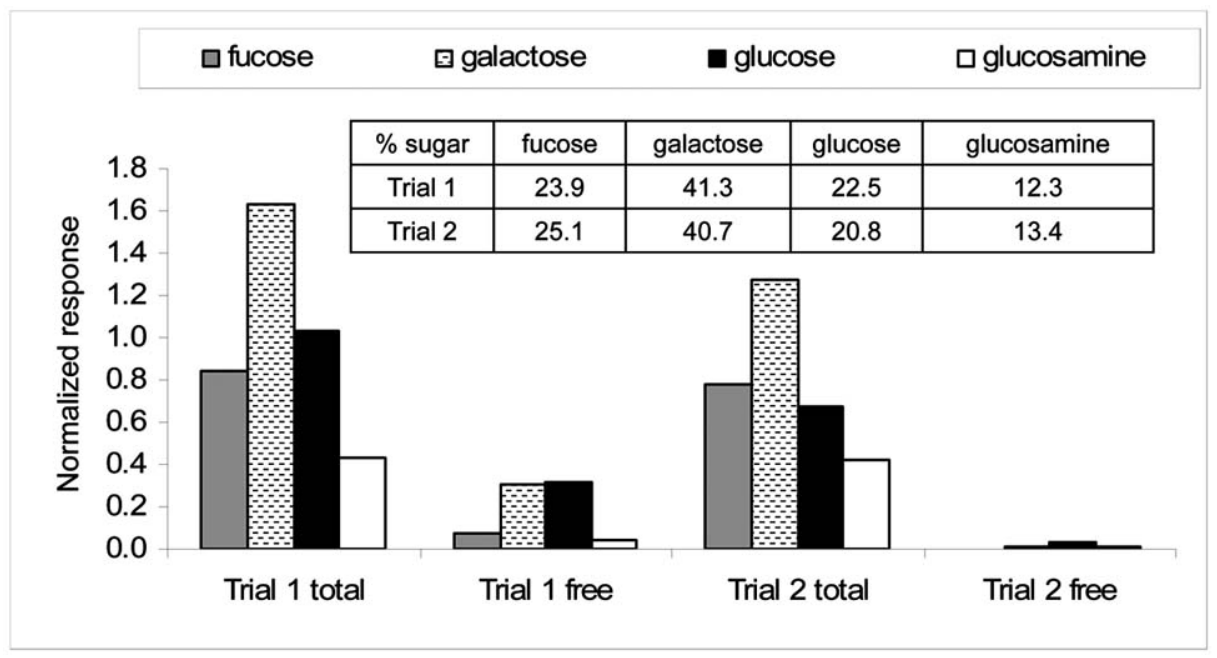

Fig. (5). Monosaccharide composition of HMO from two separate purifications determined by gas chromatography of alditol acetate derivatives. Peak areas are normalized to surrogate concentration. Free monosaccharides were measured by omitting hydrolysis step. Inset table: percent monsaccharide composition of HMO polymers from two separate trials after removing contribution of free sugars. 


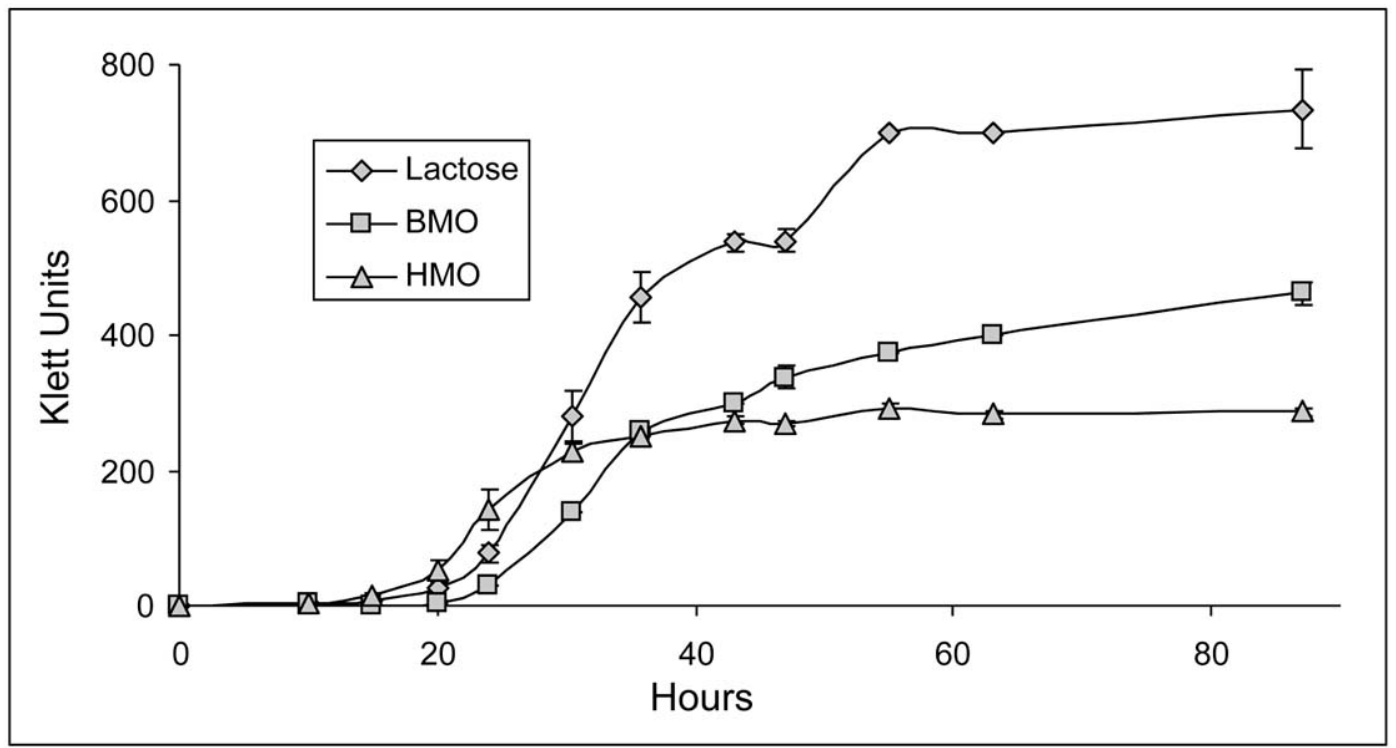

Fig. (6). Growth curves for Bifidobacterium longum biovar infantis using lactose, human milk and bovine milk oligosaccharides as the sole carbohydrate source.

comparisons. In this context, intensive biochemical characterizations of the molecules and their various bioactivities may facilitate an understanding of their importance in infant, and perhaps adult, nutrition, and may suggest individual oligosaccharide structures to target for industrial production.

To facilitate such investigations it is crucial to obtaining oligosaccharides in a pure form, free from lactose. The first time this extraction was attempted, roughly 2.5 grams were isolated from $1 \mathrm{~L}$ of breast milk. By modifying three steps from that procedure, this was increased to approximately 5.5 $\mathrm{g} / \mathrm{L}$ of milk. Although this was a substantial increase in yield, the initial concentration of HMO in the respective milks was not known. Due to the fact that HMO varies substantially across women and time of lactation, it was not possible to make a direct comparison.

While previous studies have indicated that HMO are not degraded by human digestive enzymes, the $\beta$-galactosidase used in this study was of microbial origin and may have affected the resulting purified HMO in at least two ways. There may have been some degradation of specific HMO structures by enzymatic hydrolysis, and some galactooligosaccharides (GOS) may have been formed via transglycosylation activity of the enzyme. However, in bacterial growth studies conducted with HMO purified by this method, similar HMO profiles were observed as in unprocessed milk, and masses consistent with GOS polymers were not detected [19].

The method presented here is suitable for routine preparative isolation of experimental quantities of oligosaccharides, free of contaminating lactose and monosaccharides. Furthermore, this method allows for the separation of subfractions, such as the either only neutral or only acidic HMO. This method should enable subsequent studies designed to facilitate understanding the molecular basis for the presence of these molecules in human milk.

Analysis of the monosaccharide composition of HMO from two individual purification trials indicates that the relative proportions of individual sugars was quite similar. This is perhaps surprising, as there appears to be a lot of heterogeneity in HMO composition among women during lactation, as well as between women [26], and the pooled milk used in these studies was typically contributed by fewer than 5 individual donors.

It has long been speculated that HMO may serve as fermentation substrates for intestinal microbiota, and using oligosaccharides isolated by this method it was recently shown that some strains can these polymers as an energy source $[17,20]$. One surprising feature of this activity is that $B$. longum $b v$. infantis consistently reaches higher cell densities using HMO as a substrate than other gut species, and a recent genomic analysis indicates that this bacterium contains approximately 700 unique genes many of which are specifically for this activity that other bifidobacterium strains do not possess [27]. It was of interest, therefore, to determine if oligosaccharides isolated from bovine milk using this method could support the growth of this strain. The results indicate that BMO can be fermentation substrates for microbiota, and on a mass to mass basis, are more effective than HMO. It should be kept in mind, however, that the levels in bovine milk are very low.

In sum, this method is effective in facilitating the isolation of oligosaccharides from milk, and large quantities can be produced on the bench-top. The method is reproducible across trials, and allows for the further separation of oligosaccharide fractions.

\section{ACKNOWLEDGEMENTS}

Financial support for this project was provided by the Utah Agricultural Experiment Station. This paper was approved by the Utah Agricultural Experiment Station as paper \# 8031.

\section{REFERENCES}

[1] Coppa GV, Pierani P, Zampini L, Carloni I, Carlucci A, Gabrielli O. Oligosaccharides in human milk during different phases of lactation. Acta Paediatr Suppl 1999; 88(430): 89-94. 
[2] Kunz C, Rudloff S, Baier W, Klein N, Strobel S. Oligosaccharides in human milk: structural, functional, and metabolic aspects. Annu Rev Nutr 2000; 20: 699-722.

[3] Chaturvedi P, Warren CD, Buescher CR, Pickering LK, Newburg DS. Survival of human milk oligosaccharides in the intestine of infants. Adv Exp Med Biol 2001; 501: 315-23.

[4] Gnoth MJ, Kunz C, Kinne-Saffran E, Rudloff S. Human milk oligosaccharides are minimally digested in vitro. J Nutr 2000; 130(12): 3014-20.

[5] German JB, Freeman SL, Lebrilla CB, Mills DA. Human milk oligosaccharides: evolution, structures and bioselectivity as substrates for intestinal bacteria. Nestle Nutr Workshop Ser Pediatr Prog 2008; 62: 205-22.

[6] Stahl B, Thurl S, Henker J, Siegel M, Finke B, Sawatzki G. Detection of four human milk groups with respect to Lewis-blood-groupdependent oligosaccharides by serologic and chromatographic analysis. Adv Exp Med Biol 2001; 501: 299-306.

[7] German JB, Morgan CJ, Ward RE. Milk: a model for nutrition in the 21st Century. Aust J Dairy Technol 2003; 58(2): 49-54.

[8] Morrow AL, Ruiz-Palacios GM, Jiang X, Newburg DS. Humanmilk glycans that inhibit pathogen binding protect breast-feeding infants against infectious diarrhea. J Nutr 2005; 135(5): 1304-7.

[9] Morrow AL, Ruiz-Palacios GM, Altaye M, et al. Human milk oligosaccharides are associated with protection against diarrhea in breast-fed infants. J Pediatr 2004; 145(3): 297-303.

[10] Bode L, Kunz C, Muhly-Reinholz M, Mayer K, Seeger W, Rudloff S. Inhibition of monocyte, lymphocyte, and neutrophil adhesion to endothelial cells by human milk oligosaccharides. Thromb Haemost 2004; 92(6): 1402-10.

[11] Bode L, Rudloff S, Kunz C, Strobel S, Klein N. Human milk oligosaccharides reduce platelet-neutrophil complex formation leading to a decrease in neutrophil beta 2 integrin expression. J Leukoc Biol 2004; 76(4): 820-6.

[12] Egge H, Dell A, Von Nicolai H. Fucose Containing oligosaccharides from human milk. Arch Biochem Biophys 1983; 224(1): 23553.

[13] Brand-Miller JC, McVeagh P, McNeil Y, Messer M. Digestion of human milk oligosaccharides by healthy infants evaluated by the lactulose hydrogen breath test. J Pediatr 1998; 133(1): 95-8.

[14] Sarney DB, Hale C, Frankel G, Vulfson EN. A novel approach to the recovery of biologically active oligosaccharides from milk using a combination of enzymatic treatment and nanofiltration. Biotechnol Bioeng 2000; 69(4): 461-7.

[15] Dubois M, Gilles K, Hamilton JK, Rebers PA, Smith F. A colorimetric method for the determination of sugars. Nature 1951; 168(4265): 167.
[16] York WS, Darvill AG, McNeil M, Stevenson TT. Isolation of characterization of plant cell walls and cell wall component. Method Enzymol 1985; 119: 3-30.

[17] Ward RE, Ninonuevo M, Mills DA, Lebrilla CB, German JB. In vitro fermentation of breast milk oligosaccharides by Bifidobacterium infantis and Lactobacillus gasseri. Appl Environ Microbiol 2006; 72(6): 4497-9.

[18] Redmond JW, Packer NH. The use of solid phase extraction with graphitized carbon for the fractionation and purification of sugars. Carbohydr Res 1999; 319: 74-9.

[19] LoCascio RG, Ninonuevo MR, Freeman SL, et al. Glycoprofiling of bifidobacterial consumption of human milk oligosaccharides demonstrates strain specific, preferential consumption of small chain glycans secreted in early human lactation. J Agric Food Chem 2007; 55(22): 8914-9.

[20] Ward RE, Ninonuevo M, Mills DA, Lebrilla CB, German JB. In vitro fermentability of human milk oligosaccharides by several strains of bifidobacteria. Mol Nutr Food Res 2007; 51(11): 1398405.

[21] Tao N, DePeters EJ, Freeman S, German JB, Grimm R, Lebrilla CB. Bovine milk glycome. J Dairy Sci 2008; 91(10): 3768-78.

[22] Chaturvedi P, Warren CD, Ruiz-Palacios GM, Pickering LK, Newburg DS. Milk oligosaccharide profiles by reversed-phase HPLC of their perbenzoylated derivatives. Anal Biochem 1997; 251(1): 8997.

[23] Martinez-Ferez A, Rudloff S, Guadix A, et al. Goats' milk as a natural source of lactose-derived oligosaccharides: Isolation by membrane technology. Int Dairy J 2006; 16(2): 173-81.

[24] Warren CD, Chaturvedi P, Newburg AR, Oftedal OT, Tilden CD, Newburg DS. Comparison of oligosaccharides in milk specimens from humans and twelve other species. Adv Exp Med Biol 2001; 501: 325-32.

[25] Kunz C, Rudloff S, Schad W, Braun D. Lactose-derived oligosaccharides in the milk of elephants: comparison with human milk. $\mathrm{Br}$ J Nutr 1999; 82(5): 391-99.

[26] Ninonuevo MR, Perkins PD, Francis J, et al. Daily variations in oligosaccharides of human milk determined by microfluidic chips and mass spectrometry. J Agric Food Chem 2008; 56(2): 618-26.

[27] German JB, Freeman SL, Lebrilla CB, Mills DA. Human milk oligosaccharides: evolution, structures and bioselectivity as substrates for intestinal bacteria. Nestle Nutr Workshop Ser Pediatr Prog 2008; 62: 205-18; discussion 218-22.

(C) Robert E. Ward; Licensee Bentham Open.

This is an open access article licensed under the terms of the Creative Commons Attribution Non-Commercial License (http://creativecommons.org/licenses/by-nc/3.0/) which permits unrestricted, non-commercial use, distribution and reproduction in any medium, provided the work is properly cited. 